July 7. The combined interstellar absorption in our galaxy and in NGC4254 is expected to be of the order of 0.5 magnitudes at optical wavelengths and is probably negligible in the 1.5 to $6 \mathrm{keV} \mathrm{X}$-ray region. Thus the ratio of the X-ray to the optical luminosities of the supernova on July 7 was no more than about 16 . In comparison, this ratio for the Crab Nebula is about two after correction for interstellar absorption ${ }^{4,5}$. On the other hand, for $S c o X-1$, the X-ray luminosity exceeds the optical by more than 1,000 .

In conclusion, the upper limit of the ratio of $\mathrm{X}$-ray to optical luminosities of supernova $S N 1967 h$, at an age of less than 34 days, is about eight times the observed ratio for the Crab nebula, a supernova remnant of age $\sim 900 \mathrm{yr}$.

\section{Correlation between Solar Activity and the Brightness of Jupiter's Great Red Spot}

INTEREST in Jupiter has been stimulated by recent investigations. A correlation between solar activity likely to affect the ultraviolet radiation in the solar system and the activities of the Jovian great red spot would be of some significance for those interested in the planet. In fact, examination of the sunspot cycle and the relative brightness of the Jovian red spot reveals just such a correlation.

Fig. 1 shows the curves for both Zurich sunspot numbers and the relative brightness of the Jovian red spot between

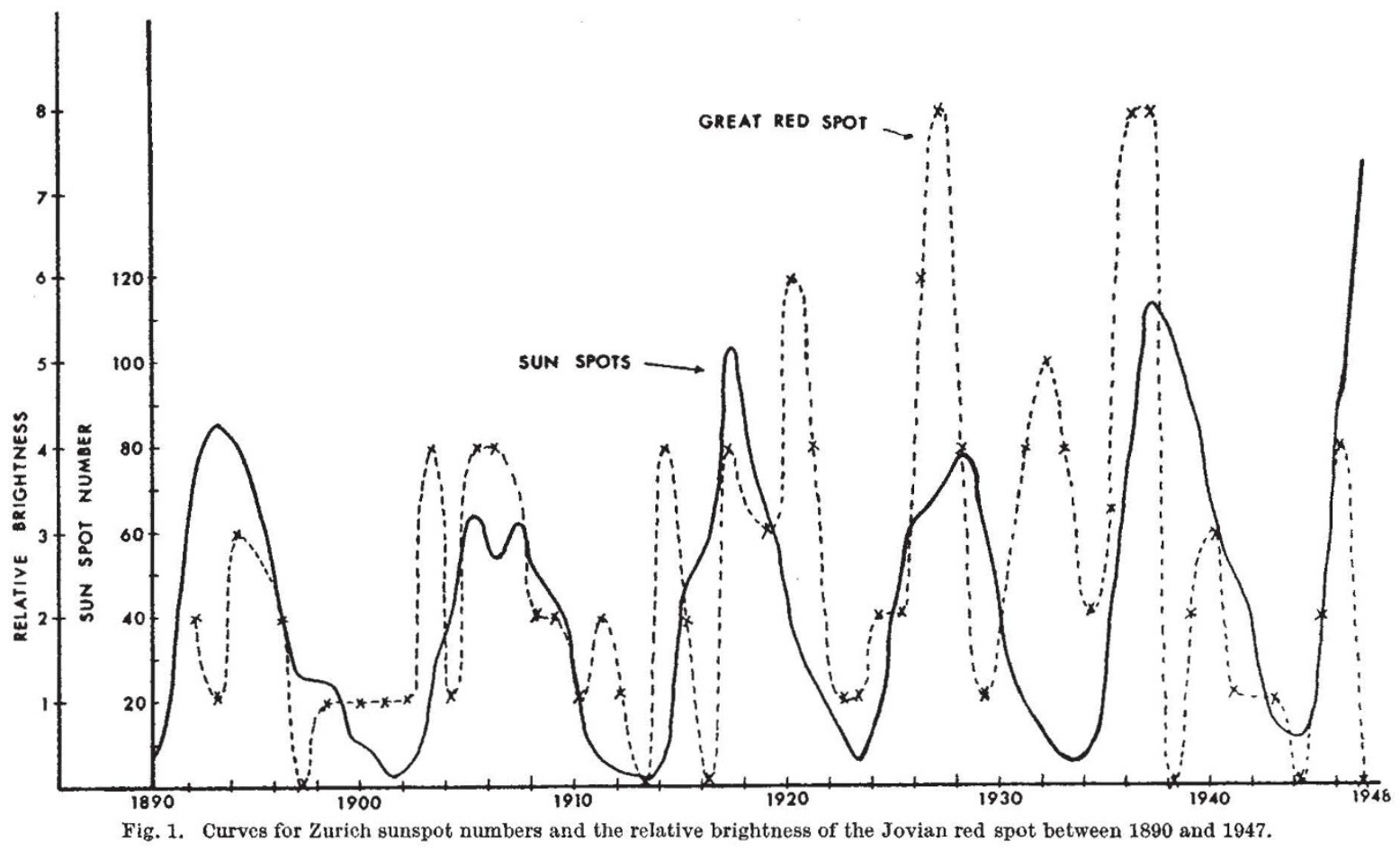

Of course, this result does not exclude the possibility of an intense burst of X-rays from $S N 1967 h$ with a decay time short compared to a few weeks.

This work was supported in part by the National Aeronautics and Space Administration.

Massachusetts Institute of Technology,

H. BRADT

S. NARANAN*

S. RAPPAPORT

Cambridge, Massachusetts.

California Institute of Technology, and

F. ZWICKY

Mt Palomar Observatory,

Pasadena, California.

Goddard Space Flight Center,

H. Ogemman†

E. BOLDT

Greenbelt, Maryland.

Received April 16, 1968.

* On leave from the Tata Institute of Fundamental Research, Bombay, India.

† National Academy of Sciences NASA postdoctoral resident research associate.

${ }^{1}$ Bradt, H., Mayer, W., Naranan, S., Rappaport, S., and Spada, G., Astrophys. J. Lett., 150, 1199 (1967).

Z Zwicky, F, IA U Circular No. 2021 (1967).

'Humason, M. L., Mayall, N. V., and Sandage, A. R., Astron. J., 61, 144 (1956).

- Bowyer, S., Byram, E. T., Chubb, T. A., and Friedman, H., Science, 146,

- O'Dell, C. R., Astrophys. J., 136, 809 (1962).
1892 and 1947. The data for the relative brightness of the red spot have been taken from Peek ${ }^{1}$ and his technique for indicating the intensity of the spot, based on observations, has been adopted.

Fig. 1 shows there is a pronounced correlation between the cyclic maxima and minima of the two curves during the period for which data were compared. In conclusion, it may be worth noting that a maximum in the current sunspot cycle is now anticipated, and also that recent observations have revealed a high intensity of the Jovian red spot.

\section{E. R. GRAF}

C. E. SMith

F. R. MCDevite

Department of Electrical Engineering,

Auburn University,

Auburn, Alabama.

Received March 1; revised April 2, 1968.

${ }^{1}$ Peek, B. M., The Planet Jupiter (Faber and Faber, London, 1958).

\section{Origin of Long-period Micropulsations}

SeVEral workers ${ }^{1}$ have discussed the origin of micropulsations in the geomagnetic field with periods of several minutes. Recently, after the discovery of the magnetospheric tail ${ }^{2}$, ideas about the origin of these micropulsa- 